Ann. Génét. Sél. anim., I972, 4 (I), 87-97.

\title{
L'HYPERTROPHIE MUSCULAIRE D'ORIGINE GÉNÉTIQUE OU CARACTÈRE CULARD ( $\left.{ }^{1}\right)$
}

\author{
B. VISSAC \\ Station de Génétique quantitative et appliquée, \\ Contre national de Recherches zootechniques, I. N.R. A., \\ 78 - Jouy-en-Josas
}

\section{RÉSUMÉ}

L'orientation de la demande du consommateur vers des carcasses de Bovins fournissant un maximum de viande maigre et tendre ainsi que le développement du croisement de $I^{r e}$ génération donnent un intérêt croissant à l'hypertrophie musculaire d'origine génétique ou caractère culard des Bovins.

La description macroscopique du caractère est maintenant assez bien connue.

- La croissance pondérale des bovins culards en général supérieure varie, par rapport aux normaux, en fonction de l'âge, du régime alimentaire et de la race ; ces animaux paraissent plus sensibles aux effets dépressifs (nutritionnels et pathologiques du milieu.)

- Le développement corporel du culard dont l'expression la plus évidente est une hypertrophie musculaire générale, se traduit également par des gradients d'hypertrophie de ce tissu : antéro-postérieur au niveau du tronc et proximal-distal au niveau des membres.

- Un net retard de maturité se manifeste sur les caractères sexuels primaires et secondaires.

Plusieurs travaux précisent la nature de l'hypertrophie musculaire. La trame conjonctive est plus lâche, le nombre total de fibres est accru. L'élimination urinaire de substances à vocation musculaire spécifique : hydroxyproline, créatine, créatinine semble perturbée.

Les auteurs s'accordent pour conclure que le caractère culard est déterminé par l'action primaire d'un couple de gènes autosomaux - action influencée par la présence de gènes modificateurs; la pénétrance varierait dans chaque race suivant que la sélection passée a été favorable ou non à la musculature.

Les objectifs de la sélection poursuivie par les éleveurs en faveur de ce caractère sont doubles :

- Sélection plutôt favorable dans les races mixtes ou à fins multiples auxquelles il permet d'acquérir rapidement une aptitude à la production de viande(Italie, Belgique, Espagne).

- Sélection très favorable en vue de constituer une souche de mâles réservés au croisement commercial.

Dans le premier cas, les effets dépressifs de l'hypertrophie musculaire sur les qualités d'élevage des femelles conduisent à rechercher des procédés objectifs efficaces de choix des mâles sur ces qualités : aptitude au vêlage, fertilité, aptitude maternelle.

Quant à la sélection d'une lignée de culards pour le croisement, son intérêt va dépendre de son coût (frais supplémentaires d'entretien d'un troupeau femelle et d'exploitation des taureaux dans le Centre d'insémination) et du revenu complémentaire qu'elle peut procurer.

(1) Rapport présenté à la Réunion annuelle de la Fédération européenne de Zootechnie, Commission de Génétique, Versailles, France; le x9 juillet I97 I. 
Le développement des recherches sur le caractère culard, dans le domaine de l'amélioration génétique tout au moins, exigerait un certain nombre d'actions communes de la part des chercheurs intéressés :

- Adoption d'un système d'appréciation uniforme et, si possible, objectif.

- Définition d'un échantillon de taureaux témoins dans chaque race où l'on exploite le caractère.

- Échange périodique d'informations et de résultats.

\section{I. - INTRODUCTION}

Depuis la guerre de I939-45 le nombre de publications consacrées à l'hypertrophie musculaire d'origine génétique des bovins ou caractère culard en Europe occidentale et aux U.S. A. a considérablement augmenté (LAUVERGNE et al., I968). L'orientation des recherches est en fait dictée par l'attitude des éleveurs de chaque pays et de chaque race vis-à-vis de ce caractère. Ses avantages en ce qui concerne la production de viande maigre et tendre, ses inconvénients relatifs aux qualités maternelles dépendent, en effet, de la demande du commerce local et des systèmes d'élevage pratiqués (dimensions des exploitations notamment). Les conséquences zootechniques de l'introduction du caractère culard dans chaque race à un instant donné, sont en outre variables suivant les aptitudes spécifiques de chacune d'elles.

Les travaux de recherche sont consacrés:

- à la description zootechnique du caractère et à l'étude de son déterminisme biologique,

— à sa transmission héréditaire et à son utilisation pour la sélection.

Les résultats de ces travaux sont rassemblés dans des études bibliographiques récentes rédigées par les chercheurs de chaque pays intéressé par la sélection de ce caractère (HANSET, I96I ; LAUVERGNE et al., I963; OLIVER et CARTWRIGHT, I968; RAIMONDI, I957 ; SOPENA QUESADA et BlanCO CACHAFEIRO, I970). Une bibliographie annotée est également rédigée et mise à jour (LAUVERGNE et al., I968).

Parallèlement à ces recherches longues et coûteuses sur bovins, les travaux relatifs à l'hypertrophie musculaire d'origine génétique effectués sur d'autres espèces plus propices à l'expérimentation comme le Porc peuvent apporter parfois d'utiles éléments d'information (SELIIER et BOCCARD, I97I). On doit à ce sujet regretter que les travaux sur l'hypertrophie musculaire chez les Ovins n'aient pas été poursuivis en Norvège, NAERLAND (I940).

\section{II. — DESCRIPTION ETT DÉTERMINISME BIOLOGIQUE DU CARAC'TÈRE}

Les recherches sur la description macroscopique des bovins culards et sur les incidences zootechniques du caractère permettent peu à peu de préciser le syndrome et ouvrent la voie à des études dynamiques du métabolisme de ces animaux.

L'avantage de croissance pondérale des veaux et jeunes bovins culards admis par 
de nombreux auteurs semble varier en fait avec le régime alimentaire, la race et l'âge. La courbe de croissance du culard est en fait caractérisée par un poids de naissance élevé, une croissance supérieure jusqu'à 6 mois-I an, et un poids adulte plus faible. Elle n'est donc pas sans intérêt pratique (VISSAC et al., I972). Les facultés d'adaptation de ces animaux à des régimes restreints (Holmes et RoBrnson, I970) ou encombrants (ANONYME, I966), à des milieux chauds semblent limitées: il serait utile de préciser ces points d'une part et d'analyser d'autre part l'efficacité alimentaire des culards dont les besoins sont probablement assez différents de ceux des bovins normaux.

Les répercussions de l'hypertrophie musculaire sur le rendement de la carcasse sont connues depuis longtemps. Les recherches récentes ont surtout précisé les gradients corporels suivant lesquels s'établit cette hypertrophie : céphalo-caudal au niveau du tronc (VISSAC, I968), distal-proximal au niveau des membres (MACKELLAR, I968), os-derme peut-être (Pomeroy et Williams, I962) et la réduction corrélative de croissance du squelette (MACKELIAR, I968; RolLINs et al., I969).

Ces caractéristiques de forme de la courbe de croissance pondérale et du développement corporel et tissulaire entraînent conjointement une disproportion entre les dimensions du canal pelvien et le volume $d u$ fotus en largeur, disproportion qui rend plus difficile les vêlages des bovins culards (DERIVAux et al., I964; FAGOT, I964; VISSAC et al., I972). L'importance des difficultés varie considérablement suivant les races en fonction de l'aptitude propre de ces dernières au vêlage. Si la pratique de la césarienne a été un élément décisif dans l'extension du caractère culard au cours des vingt dernières années, la maîtrise encore incomplète des conséquences de cette opération constitue actuellement à cet égard un frein important.

D'une façon générale, il est clair que l'hypertrophie musculaire d'origine génétique entraîne une réduction des manifestations primaires et secondaires de la sexualité tant chez le mâle que chez la femelle (Drmitropoulos, I970 ; Vissac et al., I972). I1 s'agit essentiellement d'un retard dans l'établissement de la sexualité et d'une altération du comportement de la femelle lors de l'ovulation, du vêlage ou de l'allaitement. On ne possède pas, à ce jour, d'études sur la production d'hormones hypophysaires notamment et sur la sensibilité ovarienne ou testiculaire de ces animaux, études qui permettraient de préciser les observations ci-dessus. Les résultats sur la fertilisation des ovules, sur la fécondance du sperme (Dimitropoulos, I970), sur la viabilité des embryons sont également limités et fragmentaires. Ces recherches seraient pourtant fondamentales dans la mesure où la sélection en faveur de ce caractère entraîne la production de génotypes non viables. En fait, la mortalité élevée à la naissance et qui est liée tant à la vitalité plus faible des veaux culards (PACI, I935) qu'à la présence d'anomalies cardiaques bien précises (VISSAC et al., I972) pourrait être associée à une perte d'embryons ou de fotus à divers stades de la gestation.

De nombreuses recherches ont été entreprises au cours des ro dernières années dans le but de déterminer la nature et les caractérisiques de l'hypertrophie musculaire ainsi que les phénomènes métaboliques qui lui sont associés. Si les résultats obtenus par la voie histologique ou biochimique (teneurs en DNA, RNA du muscle frais) varient en fonction du degré d'hypertrophie pondérale du (des) muscle (s) retenu (s) et de l'âge (Ouhayoun et ArNal, I969), il apparaît cependant que cette hypertrophie s'explique essentiellement par une augmentation du nombre total de fibres (Ashmore et Robinson, I969; Ouhayoun et BEAumont, I968; SMith, I949) qui 
porterait surtout sur des fibres blanches à activité glycolytique et de taille supérieure à celles de même type des animaux normaux. Les fibres à métabolisme anaérobie seraient, elles, peu affectées dans leur taille et leur nombre (BEITZ et al., I969 ; Hor,MEs et ASHMORE, I970). Ce résultat expliquerait les différences de coloration du muscle. La trame conjonctive des bovins culards est plus lâche, délimitant des myoskènes de plus grande taille (BOCCARD et al., I969). Il en résulte des teneurs du muscle en collagène et hydroxyproline plus faibles (BOCCARD, I964; LAWRIE et al., I964; HANSET, I967) : l'excrétion urinaire de cette dernière est accentuée, conséquence soit d'une dégradation accélérée du collagène, soit d'un défaut d'incorporation de la proline au cours de la collagènogenèse, conduisant à un collagène de structure physicochimique différente, en particulier plus soluble (BocCARD et al., I969). Ces particularités du tissu musculaire ne correspondent pas à un changement dans l'activité de synthèse des protéines, lipides ou glucides dans le foie (BEITz et al., x969), 1'aminoacidémie est par ailleurs comparable pour les 2 types d'animaux (HANSET, I967). Des études ont porté en outre sur la créatine et la créatinine, substances à vocation musculaire spécifique : la créatinémie des culards est très abaissée alors que leur créatininémie est nettement plus élevée ; ces différences qui se retrouvent dans le taux d'excrétion urinaire de ces substances traduisent un écart profond dans la forme du métabolisme de la créatine chez ces 2 types d'animaux (HAnser, I967). Compte tenu des particularités de structure microscopique du néphron des bovins culards (OuHayoun et ARNAL, I 969), il n'est pas interdit de penser enfin que ces animaux aient un comportement spécifique vis-à-vis du pouvoir de filtration de certains éléments sanguins.

La poursuite de ces études descriptive est fondamentale pour que l'on puisse connaître l'intérêt ainsi que les conditions d'élevage et d'utilisation optima des bovins culards. Elles devraient être complétées par des études économiques de synthèse ; les caractéristiques de ces animaux conduisent les éleveurs à envisager en effet des types d'élevage et de production différents de ceux pratiqués avec les Bovins normaux en ce qui concerne les âges de commercialisation des mâles et de réforme des femelles. Une telle orientation devrait être motivée objectivement. Sur le plan génétique il serait urgent que ces recherches descriptives nous fournissent une méthode objective et simple de mesure de ce caractère sur l'animal vivant en vue d'analyser ses variations dans les populations animales.

\section{III. - TRANSMISSION HÉRÉDITAIRE ET SÉLECTION DU CARACTÈRE}

Toutes les études génétiques reposent en fait sur des appréciations subjectives de la conformation, variables suivant les auteurs : les uns jugeant de façon générale le degré de musculature, les autres s'attachant à apprécier surtout les signes spécifiques liés au caractère culard (inclinaison du bassin, sillons intermusculaires) indépendamment de la musculature ; ces observations ont été parfois codifiées grâce à l'élaboration d'une table de pointage (VISSAc et al., I97I) où à la définition de profils types (Rolilins, I967) mais ces appréciations sont insuffisantes pour estimer notamment les différences d'expressivité du caractère. 
Toutes les études effectuées à ce jour aboutissent à la conclusion suivante :

- Le caractère culard est déterminé par l'action primaire d'un couple de gènes autosomaux.

- Cette action serait récessive pour les uns (WEBER et IBSEN, I934), incomplètement dominante (LOGEAY et VISSAC, I970 ; PACI, I935 ; RAIMONDI, I957 ; SOPENA Quesada et Blanco CACHEFEIRo, I970; WriEdT, I929) ou récessive (Fischer, I953; HANSET, I967; KIDWELL et al., I952; MASON, I963; SMITH, I949) pour la plupart : le gène " culard " ne s'exprimant qu'en présence d'un ou plusieurs couples de gènes modificateurs favorables, dont la fréquence serait liée au développement musculaire des races : maximum dans les races à forte musculature, minimum dans les races laitières à fins multiples et dans les races à forte adiposité de type anglo-saxon.

I1 est en fait apparu impossible sauf dans un cas (SOPENA QUESADA et BLANCo CACHAFEIRO, I970) de distinguer phénotypiquement l'animal hétérozygote culard de l'animal normal présentant des gènes modificateurs favorables : l'action de ce complexe génétique n'étant en pratique pas dissociable dans la sélection des animaux ; ce problème est pourtant capital dans les pays d'élevage extensif où le gène responsable du caractère est considéré comme absolument indésirable (U. S. A.).

Il faut souligner que toutes les études réalisées à ce jour ne portent que sur I à 2 générations au plus de parents contrôlés et envisagent rarement la mortalité différentielle pré- et périnatale susceptible d'intervenir entre génotypes. Leur conclusion ne peut donc être considérée d'une façon absolue.

Ce caractère ne semble pas associé à une anomalie caryologique visible : une observation sur la fréquence supérieure de cellules somatiques polyploïdes qu'il aurait été intéressant de relier à 1'hyperplasie des fibres musculaires n'a pas été confirmée depuis (POPEscu, I968).

Sur le plan de la sélection des populations bovines, les objectifs des éleveurs désirant exploiter ce caractère se ramènent à 2 catégories :

Io Sélection plutôt favorable dans des races à fins multiples ou à double fins exploitées dans de petites fermes qui ne sont plus utilisées pour la traction et qui sont concurrencées pour la production de lait par les races spécialisées ou à aptitude laitière dominante. Cette sélection permet à ces races d'acquérir rapidement une aptitude à la production de viande comparable à celle des races spécialisées (cas de la Piémontaise en Italie, de la race Haute et Moyenne Belgique, de la Blonde d'A quitaine en France de 1'Asturienne en Espagne). Il s'agit donc de la sélection en vue d'un élevage en race pure.

$2^{\circ}$ Sélection très favorable et constitution de souches de bovins culards destinées à fournir des mâles pour le croisement commercial (sud-ouest de la France). L'extension de l'insémination artificielle et la constitution de troupeaux de vaches allaitantes entretenues en feed-lot risquent en effet de favoriser la mise en place de schémas de croisements planifiés utilisant des reproducteurs culards.

\section{I. - Sélection en vue d'un élevage de race pure}

Cette sélection pratiquée dans plusieurs races européennes (Piémontaise, Haute et Moyenne Belgique, Charolaise, Blonde d'Aquitaine) consiste à retenir des animaux de type plutôt intermédiaire (VISSAC et LAUVERGNE, I969); le degré d'hypertrophie des reproducteurs choisis, variable suivant les races, est en moyenne plus accusé chez les 
mâles sélectionnés que chez les femelles dont les caractéristiques maternelles supportent l'essentiel des inconvénients liés au caractère culard (fertilité, vêlage, lactation). Le résultat de ce choix, favorable suivant des degrés variables avec la race et le sexe à un génotype hétérozygote est de maintenir ou d'accroître la fréquence du caractère culard dans la population ainsi que ses effets détériorateurs sur les qualités d'élevage des femelles (Dimitropoulos, I970; Hanset, r970).

On manque en fait d'un critère de sélection de ces qualités, opposées à la musculature, aussi efficace que celui que 1'on applique en faveur de cette dernière. Leur amélioration dans le cas présent ne peut provenir pratiquement que de la sélection naturelle dans les élevages.

En ce qui concerne l'aptitude au vêlage, la sélection devrait envisager simultanément les effets directs et maternels qui déterminent cette aptitude. Dans le premier cas les variations de forme de la courbe de croissance des veaux (rapport de la croissance prénatale à la croissance postnatale) qui semblent notables dans les populations d'animaux à musculature hypertrophiée devraient être considérées. Dans le second, la mesure de l'ouverture pelvienne des femelles et surtout des mâles, possible dès l'âge de $\mathrm{I}$ an, peut fournir un moyen efficace de sélection massale (VIssAC et al., I969I970).

La sélection des taureaux sur la fertilité de leurs filles ne peut être réalisée efficacement que par contrôle de la descendance femelle. Des études sont en cours en France en vue de préciser l'intérêt d'un contrôle précoce en station des lots de filles de différents taureaux (VISSAC et al., I969-I970). Dans le cas des Bovins culards dont la précocité corporelle est limitée on pourrait envisager la réalisation de ce contrôle dans des stations d'engraissement où les femelles seraient élevées intensivement avec contrôle d'œstrus et synchronisation en vue d'un vêlage à 2 ans. Les génisses non gestantes termineraient leur engraissement et seraient abattues.

\section{2. - Sélection en vue du croisement}

Cette solution envisagée dans le sud-ouest de la France est liée à l'organisation de schémas de croisement à simple ou double étage dans le cadre de troupeaux de vaches allaitantes. De nombreux travaux soulignent l'intérêt de tels schémas qui pourraient être réalisés à partir de femelles de races de montagne à fins multiples et de races à double fins éliminées progressivement de la traite et remplacées par des femelles de races laitières plus productives (VISSAC, I97I) ; ces femelles ont en général des qualités d'élevage favorables. L'utilisation d'un taureau culard est évidemment une des alternatives envisageables pour fournir le produit commercial (dernier étage du croisement). En fait l'emploi de ce type de taureau pour le $\mathrm{I}^{\mathrm{er}}$ croisement et l'élevage de femelles croisées n'est pas à exclure a priori dans la mesure où, d'une part, ces femelles ne présentent pas (récessivité incomplète) le caractère culard et ses effets détériorateurs et où, d'autre part, elles fournissent un pourcentage supérieur de veaux hypertrophiés.

Il apparaît effectivement que les taureaux culards engendrent des veaux croisés économiquement supérieurs en moyenne à ceux issus de taureaux normaux (VISSAC et $a l$. ., I97I). Les problèmes qui se posent à ce stade concernent l'intérêt et les modalités d'une souche de bovins culards en vue d'accroître la pénétrance du gène responsable (compte tenu de l'hypothèse ci-dessus) : 
- l'intérêt de la sélection va dépendre de son coût (entretien des taureaux dans les centres et du troupeau femelle de sélection) et du revenu supplémentaire qu'elle procurera : la variabilité des critères zootechniques est-elle la même entre taureaux culards d'une part, entre taureaux normaux d'autre part? (VISSAC et al., I'g7I).

— Les modalités de la sélection vont concerner le type de sélection adopté : sélection massale ou sur descendance croisée, le choix du lot de femelles à féconder pour ce testage en fonction de leur musculature, les modalités de constitution des lignées mâles : une lignée spécialisée par race ou une seule lignée pour l'ensemble des races. L'objectif de sélection des mâles culards est également à définir : il apparaît que l'aptitude à la naissance des veaux croisés pourrait être améliorée en sélectionnant la lignée mâle sur la forme de la courbe de croissance (voir ci-dessus) et sur la morphologie. De même le choix des taureaux destinés à être utilisés pour la production de viande rouge ou blanche devra être probablement envisagé de façon séparée. Des études sont en cours pour analyser ces différents problèmes liés à l'utilisation du caractère culard pour le croisement commercial à partir des races charolaise et blonde d'Aquitaine (VISSAC et al., I97I).

Compte tenu du coût de ces opérations de sélection soit en race pure, soit en vue du croisement, il est enfin fondamental d'en préciser les critères de rentabilité optimum suivant des techniques analogues à celles utilisées par ailleurs (HrNks, I970).

\section{IV. - CONCLUSION}

Des recherches aussi complexes, polymorphes et coûteuses que celles qui sont en cours sur l'hypertrophie musculaire d'origine génétique nécessiteraient une collaboration étroite entre chercheurs. Les problèmes les plus plus urgents concernent selon nous :

- l'adoption d'un système commun d'appréciation et si possible de mesure objective du caractère culard,

- la définition d'échantillons de taureaux de chaque race présentant le caractère culard et dont le sperme pourrait être disponible dans les pays concernés en vue de réaliser les études sur ce caractère. Étant donné le rythme rapide de changement de la musculature des animaux sous l'effet de la sélection ces échantillons devraient, pour garder leur représentativité, être renouvelés par fraction chaque année,

- l'échange d'informations et de résultats entre le groupe de chercheurs travaillant sur ce caractère est nécessaire dans des études aussi longues.

Il convient enfin de souligner encore une fois l'importance économique de ces travaux pour préciser le devenir de plusieurs populations animales qui risquent de disparaître sous l'effet d'une sélection rapide et empirique dictée souvent par la recherche du seul profit immédiat en faveur de l'hypertrophie musculaire. L'orientation de la production de viande vers le croisement entre lignées spécialisées donne, par ailleurs, un intérêt nouveau à ce caractère passionnant pour le zootechnicien comme cas limite de la production de viande et pour le généticien en raison de son déterminisme monofactoriel. 
SUMMARY

\section{HEREDITARY MUSCULAR HYPERTROPHY OR DOUBLE-MUSCLING}

The trend in consumer demand towards cattle carcases with a maximum of lean, tender meat, as well as the development of commercial crossing, are factors which enhance the significance of muscular hypertrophy as a genetic character in cattle, commonly known as double-muscling.

The macroscopic dscription of this character is by now fairly well known.

- The growth rate of double-muscle cattle is generally higher than that of norm alanimals, but it varies with age, diet and race. In addition, such animals seem particularly sensitive to unfavourable environment circumstances (diet, diseases).

- The body development of double-muscle animals clearly manifests a generalized muscular hypertrophy, but there are also certain gradients to be noted in the hypertrophy of muscle tissue : an antero-posterior gradient at the level of the trunk, and a proximal-distal gradient at the level of the limbs.

- Maturity in respect of primary and secondary sexual characteristics is clearly retarded.

There are numerous studies describing the nature of muscular hypertrophy. Connective tissue is looser in texture, and the total number of fibres is higher. Elimination in the urine of substances specifically related to muscular activity, such as hydroxyproline, creatine, creatinine, appears to be affected.

The authors conclude that the double-muscle character is determined by the primary action of a pair of autosomal genes, which is influenced by the presence of modifying genes ; the penetrance varies in each breed depending on whether previous selection has been favourable or unfavou rable to muscle development.

In selecting for this character, breeders pursue a twofold aim :

- Fairly positive selection in mixed multiple purpose breeds, to facilitate switching over from dairy to meat production (Italy, Belgium, Spain).

- Highly positive selection for the purpose of establishing a supply of males for commercial crossing purposes.

In the former case, the unfavourabe effects of muscular hypertrophy on the breeding qualities of females make it necessary to seek effective objective procedures for the selection of males in relation to the following qualities : calving capacity, fertility, maternal ability.

As regards the selection of double-muscles lines for crossbreeding purposes, the value of this exercise depends on the ratio between additional costs (in connection with the keeping of a female herd and the management of bulls in the A. I centres) and the possible additional income.

The development of research into hereditary muscular hypertrophy, at least as regards the possibilities of genetic improvement, would require some joint action on the part of interested research workers :

- Adoption of a uniform and, as far as possible, objective evaluation system.

- Definition of a reference sample of bulls for each breed in which the double-muscle character is being selected.

- Periodical exchanges of information and data.

\section{ZUSAMMENFASSUNG}

\section{MUSKULARE HYPERTROPHIE GENETISCHEN URSPRUNGS ODER DOPPELLENDEREIGENSCHAFT}

Die Orientierung der Verbrauchernachfrage nach Rinderschlachtkörpern mit einem grösstmöglichen Anteil an magerem und zartem Fleisch sowie die Entwicklung der Gebrauchskreuzung verieihen der muskulären Hypertrophie genetischen Ursprungs, allgemein Doppellendereigenschaft der Rinder genannt, ein steigendes Interesse.

Die makroskopische Beschreibung des Merkmals ist jetzt zeimlich gut bekannt.

- Die Gewichtszunahme der Doppellenderrinder ist im allgemeinen grösser als jene der 
normalen Tiere, aber sie variert je nach Alter, Futterregime und Rasse. Diese Tiere scheinen gegenüber ungünstigen Umweltbedingungen anfälliger zu sein (Ernährung, Krankheit).

- Die körperliche Entwicklung der Doppellender, die sich am deutlichsten in der allgemeinen muskulären Hypertrophie ausdrückt, erkennt man ebenfalls am Hypertrophiegefälle dieses Gewebes : Kopf-Schwanz-Gefälle auf der Höhe des Rumpfes und proximal-distal am Ansatz der Glieder.

- Die primären und sekundären geschlechtlichen Eigenschaften zeigen eine eindeutige Reifeverspätung.

In mehreren Arbeiten wird genau die Natur der muskulären Hypertrophie beschrieben. Das Bindegewebe ist schlaff, und die Gesamtzahl der Fasern ist grösser. Das Ausscheiden durch den Urin von spezifisch muskulären Substanzen, wie z. B. Hydroxyprolin, Kreatin, Kreatinin, scheint gestört zu sein.

Die Autoren stimmen überein, dass die Doppellendereigenschaft durch die primäre Wirkung von einem autosomalen Genpaar bestimmt wird. Diese Wirkung wird durch die Anwesenheit von Modifikationsgenen beeinflusst. Die Penetranz ist bei jeder Rasse unterschiedlich, je nachdem, ob die vorangegangene Selektion mehr oder weniger günstig für die Muskulatur gewesen ist.

Bei der Zuchtwahl für dieses Merkmal verfolgen die Züchter ein doppeltes Ziel :

- Ganz günstige Selektion bei den gemischten Rassen oder den Mehrzweckrassen, die aus den Milchherden herausgenommen werden und sich schnell zur Umstellung ouf Fleischproduktion eignen (Italien, Belgien, Spanien).

- Sehr günstige Selektion für die Aufstellung einer Linie von männlichen Tieren für Gebrauchs kreuzungszwecke.

Im ersten Fall führen die ungünstigen Effekte der muskulären Hypertrophie auf die Zuchtqualitäten der weiblichen Tiere dazu, nach wirksamen objektiven Methoden zur Auswahl von männlichen Tieren für folgende Qualitäten zu suchen : Abkalbungsfähigkeit, Fertilität, mütterlicher Instinkt.

Was die Selektion einer Linie von Doppellender zur Kreuzungszucht betrifft, so hängt ihr Interesse von den Kosten ab (zusätzliche Unterhaltskosten einer Herde von weiblichen Tieren und Haltung von Bullen in Stationen) und dem erzielbaren Gewinn.

Die Entwicklung der Forschungen über die Doppellender würden wenigstens im Rahmen der genetischen Verbesserung eine gewisse Anzahl gemeinsamer Vorgehen seitens der interessierten Forscher erfordern :

- Verwendung eines einheitlichen und möglichst objektiven Beurteilungssystems.

- Definierung einer Kontrollprobe von Bullen für jede Rasse, in der das Merkmal erforscht wird.

- Periodischer Austausch von Informationen und Ergebnissen.

\section{RÉFÉRENCES BIBLIOGRAPHIQUES}

Anonyme, I966. Document de travail sur les recherches françaises concernant le caractère culard Journées Étud. Féd. Eur. Zoot., Édimbourg 35 p. ronéoté.

Ashmore C. R., Robinson D. W., r969. Hereditary muscular hypertrophy in the bovine. I. Histological and biochemical characterization. Proc. Soc. exp. Biol. Med., 132, 548-554.

Beitz D. C., Young J. W., Bredahl, R. L., Jacobson N. L., Ig69. Biochemical comparisons in hypermuscled cattle. J. anim. Sci., 29, I84.

BOCCARD R., I964. Relations entre l'hypertrophie musculaire des bovins culards et le métabolisme du collagène. Annls Zootech., 13, 389-391.

Boccard R., Dumont B. L., Schmitt O., I969. Caractéristiques différentielles du tissu conjonctif des bovins normaux et "culards " (abstr.) Annls. Génét. Sél. anim., 1, i 78.

Derivaux J., Fagot V., Huet R., i964. Le problème des dystocies. Essais de pelvimétrie. Annls. Méd. vét., 8, 335-365.

Dimitropoulos E., r97o. Rapport d'activité du centre d'insémination artificielle de la province de Namur pour l'année i97o. 8 p. ronéoté.

FAGOT V., I964. La vraie conformation de boucherie en rapport avec les possibilités de vêlage. Zootechnia, 13, 3-17.

Fischer H., I953. Die Genetik der Doppellendigkeit beim Rind. Fortpfl. Besam. Haustiere, 3, 25-27.

Hanset R., I96I. Le problème des bovins " à croupe de poulain ". Annls Méd. vét., 105, 14-3I.

HANSET R., I966. Le problème de l'hypertrophie musculaire ou caractère " culard " dans la race bovine de Haute et Moyenne Belgique. Journées Étud. Féd. Eur. Zootech., Édimbourg, 2 I p., ronéoté.

HANSET R., I967. Le problème de l'hypertrophie musculaire ou caractere "culard " dans la race bovine de Moyenne et Haute Belgique. Annls Méd. vét., 111, I40-I80. 
Hinks, r970. Performance test procedures for meat production amongst dairy bulls used in A. I. Anim. Prod., 1970, 12, 577-583.

Holmes J. H. G., Ashmore C. R., I97o. Muscle fibre types in double muszled calves. abstr. J. anim. Sci., 31, I 3-184.

Holmes J. H. G., Robinson D. W., r97o. Hereditary muscular hypertrophy in the bovine : metabolic response to nutritional stress. J. anim. Sci., $\mathbf{3 1}, 776-780$.

Kidwell J. F., Vernon E. H., Crown R. M., Singletary C. B., r952. Muscular hypertrophy in cattle. J. Hered., 43, 63-68.

Lauvergne J.-J., Vissac B., Perramon A., I963. Étude du caractère culard. I. Mise au point bibliographique. Annls. Zootech., 12, I35-I56.

Lauvergne J.-J., Boyazoglu J. G., Hubert Denise., I968. Le caractère culard. Bibliographie annotée. Bull. tech. Dép. Génét. anim. (Inst. nat. Rech. agron. Fr.), (2).

Lavin Arenas S., I964. La raza Asturiana de los valles como productora de carne. Noticias Neosan,122, $6 I-87$.

Lawrie R. A., Pomeroy R. W., Williams D. R., I964. Studies in the muscles of meat animals. IV. Comparative composition of muscles from "doppelender " and normal sibling heifers. J. agric. Sci., Camb., 62, 89-92.

Logeay B., Vissac B., I97o. Étude du caractère culard. V. Expérience de croisement entre bovins culards et normaux. Annls Génét. Sél. anim., 2, 5-17.

Mackellar J. C., I968. Muscular hypertrophy in South Devon cattle. Th. presented for the Diploma of Fellowship of the Royal College of Veterinary Surgeons, Bristol, sept., $54 \mathrm{p}+$ XIII.

Mason I. L., I963. Symptoms of muscular hypertophy in heterozygous steers. Anim. Prod., 5, 57-65.

NaErland G., r940. Does the "Doppelender " condition occur in other species of domestic animals besides cattle? universal hyperplasia of the trunk and limbs in sheep. Skand. VetTidskr, 30, 8I r.

Oliver W. M., Cartwright T. C., rg68. Double muscling in cattle. A review of expression, genetics and economic implication. Texas Agr. exp. stat. Dept. Anim. Sci. tech. rep., 12, 58 p. ronéoté.

Ouhayoun J., Beaumont A., I968. Étude du caractère culard. III. Anatomie microscopique comparée du tissu musculaire de mâles charolais normaux et culards. Annls. Zootech., 17, 2I3-223.

Ouhayoun J., Arnal T., I969. Étude du caractère culard. IV. Anatomie microscopique comparée du rein de mâles charolais normaux et culards. Annls. Génét. Sél. anim., 1, ror-ıo8.

PACI C., I935. Indagini e controlli sulla formazione della sottorazza albese. Riv. Zootec., 12, I49-I54, I 59, I $97-202,207-208,240-250,255$.

Pomeroy R. W., Williams D. R., i962. Muscular hypertrophy in cattle. Anim. Prod., 4, 302 (abstr.).

Popescu, P. C., I968. Observations cytogénétiques chez les bovins charolais normaux et culards. Annls. Gênét., 11, 262-264.

Raimondi R., r957. Studio sui bovini piemontesi " a groppa doppia " Annali Accad. Agric. Torino, 99, $60 \mathrm{p}$.

RaImondi R., r96I. Esperienza comparativa di ingrassamento e di macellazione tra vitelli piemontesi e "groppa doppia " e comuni. Annali Sper. Agr., 15, I50-I5I.

RaImondi R., I963. Risultati di una prova di allevamento di bovine piemontesi del tipo "della coscia". Annali Sper. Agr., 17, 471-489.

Rollins W. C., I967. Developing a meat index for beef cattle. California Agric., 21 (5), I5-I7.

Rollins W. C., Julian L. M., Carroll F. D., rg69. A note on the body composition of a double-muscled female and a normal female from a linebred. Aberdeen Angus herd. Anim. Prod., 11, III-II4.

Sellier P., Boccard R., I97I. Étude du taux d'hydroxyproline d'un muscle et de ses relations avec certaines caractéristiques de composition corporelle dans les races Large-White et de Pietrain. Annls Génét. Sél. anim., 3, 433-448.

Sмiтн W. H., 1949. The occurence of the double-muscled character in beef cattle. M. Sci. Thesis, Kansas State College. $65 \mathrm{p}$.

Sopena Quesada A., Blanco Cachafeiro M. E., 1970. El caracter "culon " en el ganado vacuno. Zootechnia, 19, 489-499.

Sopena Quesada A., Blanco Cachafeiro M. E., I970. Transmision genetica del caracter $\alpha$ culon " en el ganado vacuno Zootechnia, 19, 501-526.

VISSAc B., I968. Étude du caractère culard. II. Incidence du caractère culard sur la morphologie générale des bovins. Annls. Zootech., 17, 77-ror.

Vissac B., Lauvergne J.-J., I969. Enquête sur le caractère culard dans la zone charolaise (abstr.) Annls Génét. Sél. anim., 1, 177.

Vissac B., Ménissier F., Perreau B., i97r. Le caractère culard et son utilisation pratique. Revue Élev., (fév), 35-50, (mars), 5 I-68.

Vissac B., Frebling J., Ménissier F., r969-I97o. Amélioration génétique des bovins pour la production de viande. Cours Approf. d'A mélior. Génét. des Anim. Domest., 2 I3 p., ronéoté.

Vissac B., I97x. L'utilisation optimale du matériel génétique. Les apports des techniques de croisement par rapport aux autres méthodes d'amélioration génétique : bovins à viande (troupeaux spécialisés). $X$ e Congr. int. de Zootech., 69-8I. 
Vissac B., Perreau B., Ménissier F., Mauléon P., 1972. Étude du caractère culard. VI. Bilan d'exploitation d'un troupeau de femelles. Annls. Génét. Sél. anim. (à paraître).

WEBER A. D., IBSEN H. L., I934. The occurence of the double-muscled character in purebred beef cattle. Proc. Am. Soc. Anim. Prod., 228- 32.

WRIEDT C., I999. Die Vererbung des Doppelender-charakters bei Rindern. Z. indukt. Abstamm-u. Vererblehre, 51, $482-486$. 